\title{
ARM Climate Research Facility Quarterly Value-Added Product Report
}

Chitra Sivaraman

August 2011

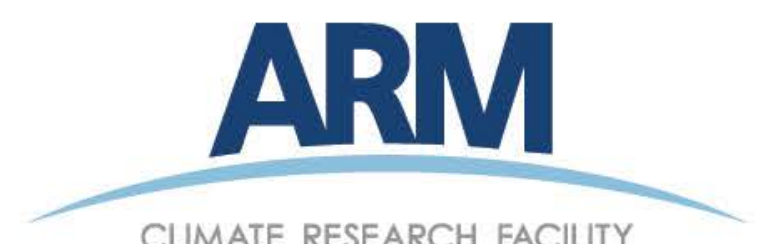




\section{DISCLAIMER}

This report was prepared as an account of work sponsored by the U.S. Government. Neither the United States nor any agency thereof, nor any of their employees, makes any warranty, express or implied, or assumes any legal liability or responsibility for the accuracy, completeness, or usefulness of any information, apparatus, product, or process disclosed, or represents that its use would not infringe privately owned rights. Reference herein to any specific commercial product, process, or service by trade name, trademark, manufacturer, or otherwise, does not necessarily constitute or imply its endorsement, recommendation, or favoring by the U.S. Government or any agency thereof. The views and opinions of authors expressed herein do not necessarily state or reflect those of the U.S. Government or any agency thereof. 


\section{ARM Climate Research Facility Quarterly Value-Added Product Report}

Third Quarter:

April 01-June 30, 2011

C Sivaraman

August 2011

Work supported by the U.S. Department of Energy,

Office of Science, Office of Biological and Environmental Research 


\section{Abstract}

The purpose of this report is to provide a concise status update for value-added products (VAP) implemented by the Atmospheric Radiation Measurement Climate Research Facility. The report is divided into the following sections: (1) new VAPs for which development has begun, (2) progress on existing VAPs, (3) future VAPs that have been recently approved, (4) other work that leads to a VAP, and (5) top requested VAPs from the archive. New information is highlighted in blue text. New information about processed data by the developer is highlighted in red text. The upcoming milestones and dates are highlighted in green. 


\section{Acknowledgements}

This report is developed largely from the information submitted by the developers and task leads to the Extraview reporting system (http://ewo.arm.gov). Special thanks to our VAP development team for providing timely and complete updates to the Engineering Change Orders and Engineering Work Orders, Dana Dupont and Rolanda Jundt, who make sure that this information is posted accurately on the ARM website, and to Stefanie Shamblin of Oak Ridge National Laboratory for providing the metrics report on VAPs. 


\section{Contents}

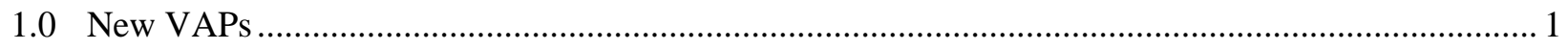

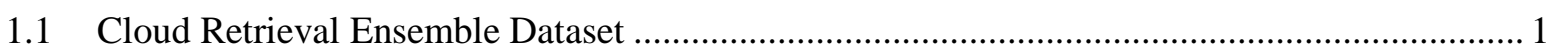

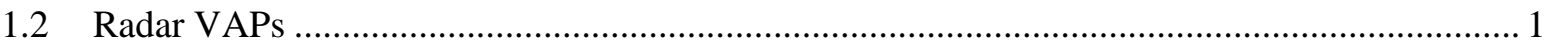

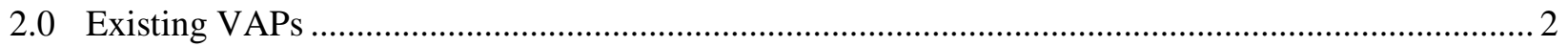

2.1 Atmospherically Emitted Radiance Interferometer Noise Filter................................................. 2

2.2 AERI Profiles of Water Vapor and Temperature................................................................... 2

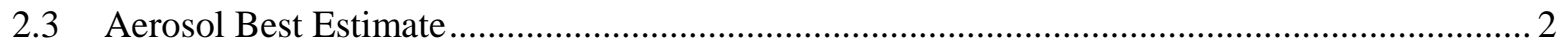

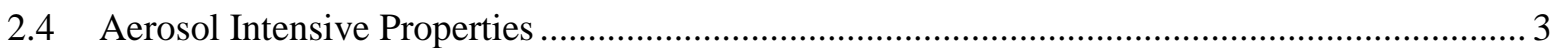

2.5 Aerosol Optical Depth Derived From Either MFRSR or NIMFR …....................................... 3

2.6 Active Remote Sensing of Clouds ................................................................................... 4

2.7 Best-Estimate Fluxes from EBBR Measurements and Bulk Aerodynamics

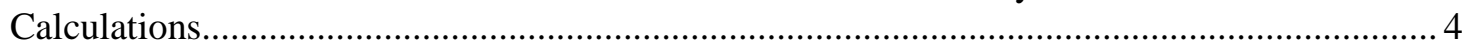

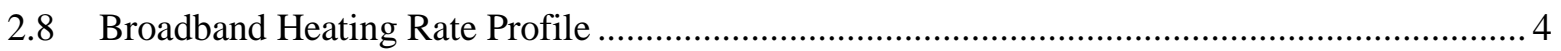

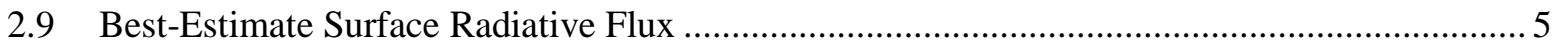

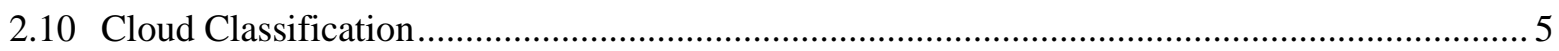

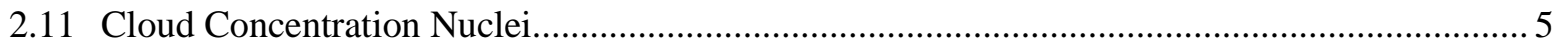

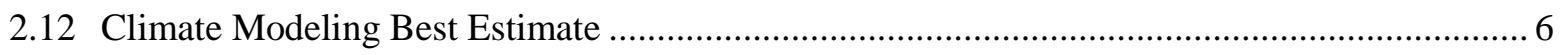

2.13 G-Band Vapor Radiometer Precipitable Water Vapor........................................................... 6

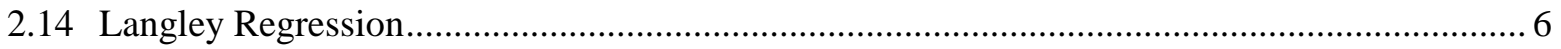

2.15 Microwave Radiometer-Scaled Sonde Profiles .............................................................. 7

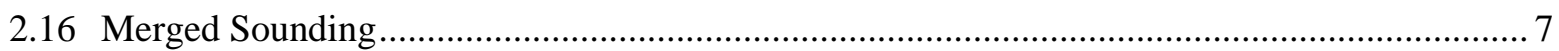

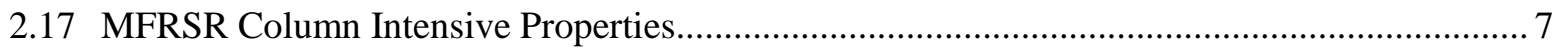

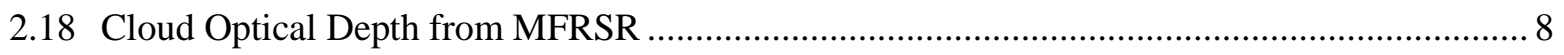

2.19 Continuous Baseline Microphysical Retrieval............................................................... 8

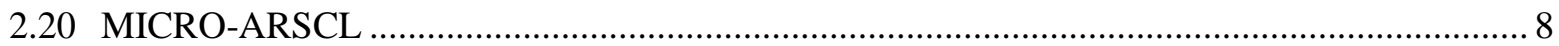

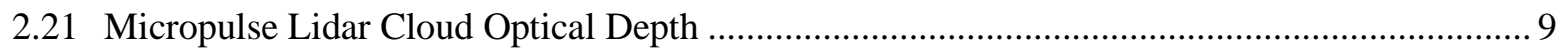

2.22 Micropulse Lidar Polarized Average ........................................................................... 9

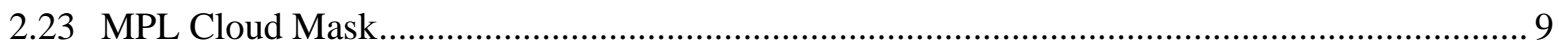

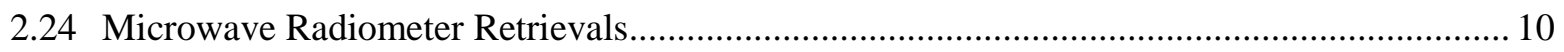

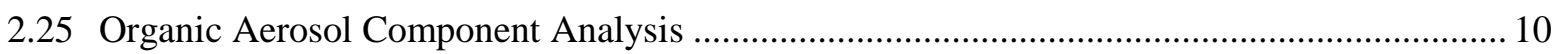

2.26 Data Quality Assessment for ARM Radiation Data ........................................................... 11

2.27 Raman Lidar Profiles_Aerosol Scattering Ratio ................................................................. 11

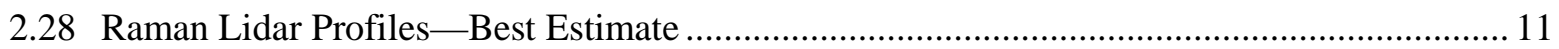

2.29 Raman Lidar Profiles_Depolarization Ratio ....................................................................... 12

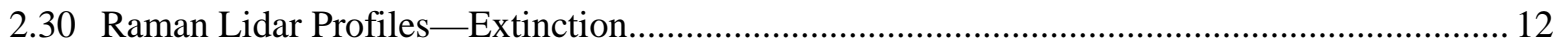




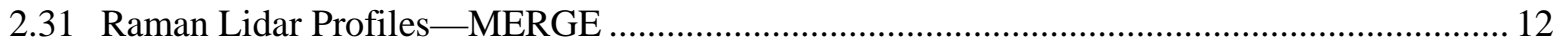

2.32 Raman Lidar Profiles_-Mixing Ratio.............................................................................. 13

2.33 Radiatively Important Parameters Best Estimate............................................................... 13

2.34 SGP Area Surface Cloud and SW Radiation Grid ............................................................ 14

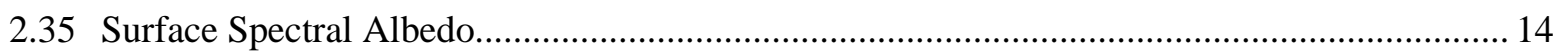

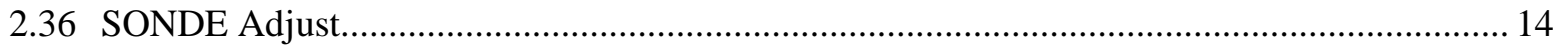

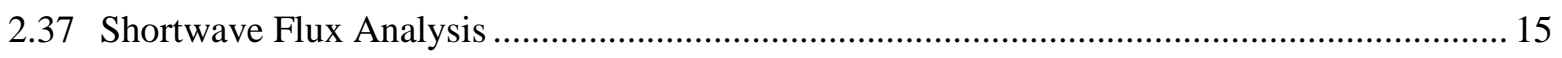

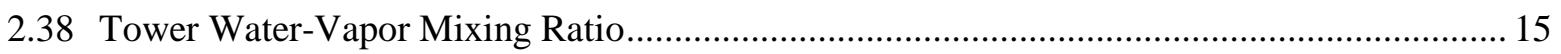

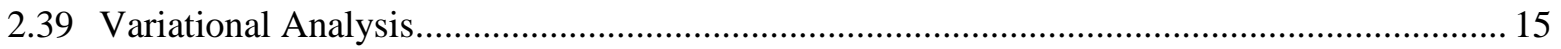

2.40 W-Band ARM Cloud Radar Active Remote Sensing of Clouds .......................................... 15

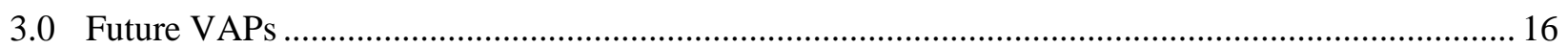

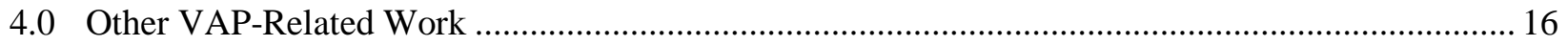

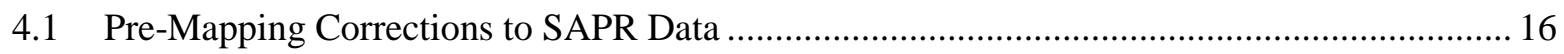

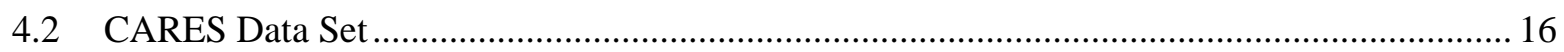

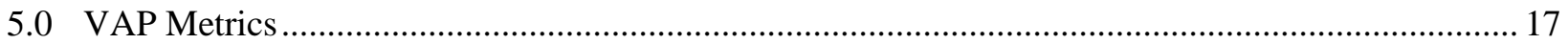




\subsection{New VAPs}

This section describes new activities that have begun in the last quarter after being approved by the ARM Infrastructure and Science Team.

\subsection{ARM Cloud Retrieval Ensemble Data Set (ACRED)}

Translator: Shaocheng Xie, Lawrence Livermore National Laboratory

Developer: Chuanfeng Zhao and Renata McCoy, Lawrence Livermore National Laboratory

Engineering Work Order-13590 has been approved to address the uncertainty in cloud retrievals and to provide three different retrievals at the five permanent ARM research sites. The implementation plan is available at https://wiki.arm.gov/bin/view/Engineering/VAPWhitePapers.

The first evaluation data set has been staged in the evaluation area. The data are currently available for the five ARM Climate Research Facility sites: SGP.C1 (Lamont, OK), NSA.C1 (Barrow, AK), TWP.C1 (Manus Island, PNG), TWP.C2 (Nauru), and TWP.C3 (Darwin, AU) in the evaluation area. For each site, ACRED contains three to six retrieval products.

Review feedback provided by users.

\subsection{Radar VAPs}

Translator: Scott Collis, Argonne National Laboratory

Developer: Scott Collis, Argonne National Laboratory

Engineering Change Order-00796 was approved to initiate and coordinate the development of the VAP generation procedures associated with data produced by the Recovery Act-funded precipitation radars.

Real-time displays and analysis systems were deployed and used at the Southern Great Plains (SGP) Central Facility as part of the Midlatitude Continental Convective Clouds Experiment with great success (very positive feedback from National Aeronautics and Space Administration [NASA] and Department of Energy [DOE] principal investigators). These systems continue to be operational.

An initial draft of an implementation plan for a Cartesian-mapped moments VAP has been completed. 


\subsection{Existing VAPs}

This section describes the status of each VAP and the ongoing activities that were approved to improve the performance of or maintain existing VAPs. The information is abstracted primarily from the monthly updates provided by the development team to the ECOs.

\subsection{Atmospherically Emitted Radiance Interferometer Noise Filter (AERINF)}

Translator: Sally McFarlane, Pacific Northwest National Laboratory

Developer: Tim Shippert, Pacific Northwest National Laboratory

Status: Stopped

Tier: Production

Engineering Change Order-00449 has been approved to run AERINF at the ARM Mobile Facility (AMF) sites and adapt to the new Recovery Act-funded AERIs.

No progress has been made due to higher priorities. The VAP has stopped producing data due to a software problem with the input summary files.

Next milestone: Release a new AERINF product to run at AMF sites by December 31, 2011.

\subsection{AERI Profiles of Water Vapor and Temperature (AERIPROF)}

Translator: Sally McFarlane, Pacific Northwest National Laboratory

Developer: Tim Shippert, Pacific Northwest National Laboratory

Status: Operational

Tier: Production

There are no ECOs for this VAP.

\subsection{Aerosol Best Estimate (AEROSOLBE)}

Translator: Connor Flynn, Pacific Northwest National Laboratory

Developer: Annette Koontz, Pacific Northwest National Laboratory

Status: In Development

Tier: Evaluation 
Engineering Change Order-00360 was approved to update the VAP to add logic to capture red and blue wavelength quantities, update the quality check fields, and use the latest Aerosol Intensive Properties (AIP) data. The VAP was estimated to be released by June 1, 2011.

Updates to the quality control (QC) fields and source flags were completed, and the data were provided to the Data Quality Office for review. The Data Quality office has provided a review of the data. The second draft of the technical report has been completed. This task is now waiting for translator approval on documentation and quality check feedback. No update has been provided on the progress of the task.

Next milestone: Translator reviews the product, and product is released. The milestone has been pushed back to September 30, 2011.

\subsection{Aerosol Intensive Properties (AIP)}

Translator: Connor Flynn, Pacific Northwest National Laboratory

Developer: Annette Koontz, Pacific Northwest National Laboratory

Status: In Development

Tier: Production

Engineering Change Order-00363 was approved to update the VAP with various changes. Please see the ECO for details. After the VAP was released, the Data Quality Office provided a review of the data.

Based on the review from the Data Quality Office, some corrections were made to the quality checks. The VAP is waiting to be reviewed by the translator. No update has been provided on the progress of the task.

Next milestone: Release the product for routine processing at the Data Management Facility (DMF). The milestone has been pushed back to October 01, 2011.

\subsection{Aerosol Optical Depth Derived From Either MFRSR or NIMFR (AOD)}

Translator: Connor Flynn, Pacific Northwest National Laboratory

Developer: Connor Flynn, Pacific Northwest National Laboratory

Status: Operational

Tier: Production

Engineering Change Order-00089 was approved to run the AOD VAP at the AMF sites.

The Io values were provided for AMF at Germany, and the data have been processed. 
The processed data have been placed in the evaluation area for Germany.

Next milestone: Provide Io values, and process data from Point Reyes, Azores, and China by September 15, 2011.

\subsection{Active Remote Sensing of Clouds (ARSCL)}

Translator: Mike Jensen, Brookhaven National Laboratory

Developer: Karen Jones, Pacific Northwest National Laboratory

Status: Operational

Tier: Production

Engineering Change Order-00086 has been approved to catch up on processing of ARSCL data and development of new ARSCL for the upgraded KAZR system.

Due to higher ingest priorities, the development of new ARSCL for the upgraded KAZR system has not begun.

Data have been processed for TWP-C1 for July through November 2009.

Next milestone: Complete the implementation plan for the KAZR VAP and provide evaluation data by the working group meeting in September 2011.

\subsection{Best-Estimate Fluxes from EBBR Measurements and Bulk Aerodynamics Calculations (BAEBBR)}

Translator: Shaocheng Xie, Lawrence Livermore National Laboratory

Developer: Krista Gaustad, Pacific Northwest National Laboratory

Status: Operational

Tier: Production

There are no open ECOs for this VAP.

\subsection{Broadband Heating Rate Profile (BBHRP)}

Translator: Sally McFarlane, Pacific Northwest National Laboratory

Developer: Tim Shippert, Pacific Northwest National Laboratory

Status: Operational 
Tier: Evaluation

Engineering Change Order-00219 has been approved to make updates to the BBHRP/Radiatively Important Parameters Best Estimate (RIPBE) interface, run the alpha version, analyze data, prepare a technical report, and deliver data to the evaluation area.

The BBHRP runs at SGP have been completed.

Next milestone: Run BBHRP with reprocessed RIPBE (using the latest MICROBASE and AEROSOLBE data), and release the data.

\subsection{Best-Estimate Surface Radiative Flux (BEFLUX)}

Translator: Sally McFarlane, Pacific Northwest National Laboratory

Developer: Yan Shi, Pacific Northwest National Laboratory

Status: Operational

Tier: Production

There are no open ECOs for this VAP.

\subsection{Cloud Classification (CLDCLASS)}

Translator: Sally McFarlane, Pacific Northwest National Laboratory

Developer: Chaomei Lo, Pacific Northwest National Laboratory

Status: No Development

Tier: Evaluation

There are no open ECOs for this VAP.

\subsection{Cloud Concentration Nuclei (CCN)}

Translator: Sally McFarlane, Pacific Northwest National Laboratory

Developer: Chitra Sivaraman, Pacific Northwest National Laboratory

Status: In Development

Tier: Evaluation

Engineering Change Order-00813 has been approved to begin implementation of the Ghan CCN Retrieval algorithm, develop a version of the code, provide one month of data for review, and add quality checks. 
Quality checks have been added to the product and analyzed. Two months of data have been provided to the user for review. The Technical Report has been completed.

Next milestone: Release two years of data to the evaluation area, and begin evaluating the data with aircraft data before the working group meeting in September 2011.

\subsection{Climate Modeling Best Estimate (CMBE)}

Translator: Shaocheng Xie, Lawrence Livermore National Laboratory

Developer: Renata McCoy, Lawrence Livermore National Laboratory

Status: Operational

Tier: Evaluation

Engineering Change Order-00620 has been approved to make updates to run SGP, North Slope of Alaska (NSA) and Tropical Western Pacific (TWP) sites, publish CMBE to the Earth System Federated Grid (ESFG), and adhere CMBE to ARM data object design (DOD) standards to produce ARMBE.

The evaluation of CMBE update for SGP is almost complete. Addition of clear-sky radiative fluxes is in progress.

Next milestone: Release enhanced version of CMBE at SGP and release ARMBE in ESFG and meet ARM DOD Standards by September 30, 2011.

\subsection{G-Band Vapor Radiometer Precipitable Water Vapor (GVRPWV)}

Translator: Connor Flynn, Pacific Northwest National Laboratory

Developer: Annette Koontz, Pacific Northwest National Laboratory

Status: Operational

Tier: Production

There are no open ECOs for this VAP.

\subsection{Langley Regression (LANGLEY)}

Translator: Connor Flynn, Pacific Northwest National Laboratory

Developer: Annette Koontz, Pacific Northwest National Laboratory

Status: Operational

Tier: Production 
There are no open ECOs for this VAP.

\subsection{Microwave Radiometer-Scaled Sonde Profiles (LSSONDE)}

Translator: Connor Flynn, Pacific Northwest National Laboratory

Developer: Annette Koontz, Pacific Northwest National Laboratory

Status: Operational

Tier: Production

There are no open ECOs for this VAP.

\subsection{Merged Sounding (MERGESONDE)}

Translator: Mike Jensen, Brookhaven National Laboratory

Developer: David Troyan, Brookhaven National Laboratory

Status: In Development

Tier: Evaluation

Engineering Change Order-00092 has been approved to add quality check fields, release the first version of the code to the DMF, and provide version 2 of the data to evaluation.

The VAP is almost ready to be released to the DMF after meeting DOD and QC standards. The second version of MERGESONDE has also been ported to engineering.

Next milestone: Release the product for routine processing at the DMF. Review feedback provided by the users on version 2 of the MERGESONDE VAP.

\subsection{MFRSR Column Intensive Properties (MFRSRCIP)}

Translator: Connor Flynn, Pacific Northwest National Laboratory

Developer: Annette Koontz, Pacific Northwest National Laboratory

Status: In Development

Tier: Evaluation

Engineering Change Order-00823 has been approved to develop a VAP to retrieve aerosol column intensive properties from the multifilter rotating shadowband radiometer (MFRSR), including single scattering albedo, asymmetry parameter, and bi-modal log-normal size distributions. 
No progress has been made due to problems in installing libraries and licenses needed to run the code developed by the mentor.

Next milestone: Address problems with the libraries and produce evaluation data by working group meeting in September 2011.

\subsection{Cloud Optical Depth from MFRSR (MFRSRCLDOD)}

Translator: Sally McFarlane, Pacific Northwest National Laboratory

Developer: Yan Shi, Pacific Northwest National Laboratory

Status: Operational

Tier: Production

Engineering Change Order-00287 has been approved to update the VAP to run with MWRRET as input, run and evaluate data at Azores, and release the product.

The VAP needed to be reviewed again due to changes in Io units in one of the input datastreams. The VAP has been automated to deal with unit changes over time.

Next milestone: Release the product for routine processing at DMF, and reprocess historical data.

\subsection{Continuous Baseline Microphysical Retrieval (MICROBASE)}

Translator: Mike Jensen, Brookhaven National Laboratory

Developer: Maureen Dunn, Brookhaven National Laboratory

Status: Operational

Tier: Production

Engineering Change Order-00804 has been approved to update the VAP with quality checks and release to production.

The Technical Report has been completed. The VAP is being adapted to read mwrret.c2 and mergesonde.c2 and is ready to be released. Progress has been made to process the data at SGP for 20022006 as input to RIPBE.

Next milestone: Release the VAP to use mwrret.c2 for routine processing at the DMF, and reprocess TWP data,

\subsection{MICRO-ARSCL (MICROARSCL)}

Translator: Mike Jensen, Brookhaven National Laboratory 
Developer: Ed Luke, Brookhaven National Laboratory

Status: In Development

Tier: Evaluation

Engineering Change Order-00847 has been approved to solve the spectral imaging problem and porting MICROARSCL to the ARM computer cluster at Oak Ridge National Laboratory.

Porting the code to Oak Ridge National Laboratory has not begun due to hardware problems. The task with spectral imaging has been $60 \%$ completed.

Next milestone: Reprocess historical data at Oak Ridge National Laboratory. This milestone has been pushed back to October 30, 2011.

\subsection{Micropulse Lidar Cloud Optical Depth (MPLCOD)}

Translator: Sally McFarlane, Pacific Northwest National Laboratory

Developer: Chaomei Lo, Pacific Northwest National Laboratory

Status: No development

Tier: Evaluation

There are no open ECOs for this VAP.

\subsection{Micropulse Lidar Polarized Average (MPLAVG)}

Translator: Connor Flynn, Pacific Northwest National Laboratory

Developer: Annette Koontz, Pacific Northwest National Laboratory

Status: Operational

Tier: Production

There are no open ECOs for this VAP.

\subsection{MPL Cloud Mask (MPLCMASK)}

Translator: Sally McFarlane, Pacific Northwest National Laboratory

Developer: Chitra Sivaraman, Pacific Northwest National Laboratory

Status: Operational 
Tier: Production

Engineering Change Order-00778 is approved to process historical data.

Historical data have been processed and archived for the SGP site from May 11, 2004, to present. Data have been processed and archived for AMF sites Germany and China.

Next milestone: Process historical data at TWP, SGP (1999-2004), and NSA.

\subsection{Microwave Radiometer Retrievals (MWRRET)}

Translator: Sally McFarlane, Pacific Northwest National Laboratory

Developer: Krista Gaustad, Pacific Northwest National Laboratory

Status: Operational

Tier: Production

Engineering Change Order-00526 has been approved to transition the product from evaluation to production, release the product, and process historical data.

This ECO is 100\% complete. The VAP has been released to production, and historical data have been processed and archived.

Next milestone: Start routine processing of data at the DMF.

\subsection{Organic Aerosol Component Analysis}

Translator: Jerome Fast, Pacific Northwest National Laboratory

Developer: Tim Shippert, Pacific Northwest National Laboratory

Status: In Development

Tier: Evaluation

Engineering Change Order-00838 has been approved to develop a VAP to estimate organic aerosol components from Aerosol Mass Spectrometers (AMS) and Aerosol Chemical and Speciation Monitors (ACSM) to be deployed at ARM's sites and as part of the Mobile Aerosol Observing System (MAOS).

Progress has been made to install the positive matrix function (PMF) commercial off-the-shelf (COTS) software and run the software on Unix and Linux machines using the Wine software library. 


\subsection{Data Quality Assessment for ARM Radiation Data (QCRAD)}

Translator: Sally McFarlane, Pacific Northwest National Laboratory

Developer: Yan Shi, Pacific Northwest National Laboratory

Status: Operational

Tier: Production

Engineering Change Order-00247 has been approved to run the VAP with Azores data, fix quality check problems, and analyze data.

Quality checks additions have been completed, and the VAP has been released to the DMF.

\section{Next milestone: Reprocess historical data.}

\subsection{Raman Lidar Profiles-Aerosol Scattering Ratio (RLPROFASR)}

Translator: Connor Flynn, Pacific Northwest National Laboratory

Developer: Chitra Sivaraman, Pacific Northwest National Laboratory

Status: Operational

Tier: Production

Engineering Change Order-13736 has been approved to make changes to the VAP to run with the new Raman Lidar at Darwin.

No progress has been made due to other priorities.

Next milestone: Release VAP to run at TWP, and begin routine processing at the DMF by September 30, 2011.

\subsection{Raman Lidar Profiles-Best Estimate (RLPROFBE)}

Translator: Connor Flynn, Pacific Northwest National Laboratory

Developer: Chitra Sivaraman, Pacific Northwest National Laboratory

Status: In Development

Tier: Production

Engineering Change Order-00106 has been approved to update the RLPROFBE VAP to input the new release of all its input data, add quality checks, and release the VAP. 
The VAP is ready to be released.

Next milestone: Release VAP and process historical data. Complete technical report and update web pages by October 01, 2011.

\subsection{Raman Lidar Profiles-Depolarization Ratio (RLPROFDEP)}

Translator: Connor Flynn, Pacific Northwest National Laboratory

Developer: Chitra Sivaraman, Pacific Northwest National Laboratory

Status: Operational

Tier: Production

Engineering Change Order-13736 has been approved to make changes to the VAP to run with the new Raman Lidar at Darwin.

No progress has been made due to other priorities.

Next milestone; Release VAP to run at TWP, and begin routine processing at the DMF by September 30, 2011.

\subsection{Raman Lidar Profiles-Extinction (RLPROFEXT)}

Translator: Connor Flynn, Pacific Northwest National Laboratory

Developer: Chitra Sivaraman, Pacific Northwest National Laboratory

Status: Operational

Tier: Production

Engineering Change Order-13736 has been approved to make changes to the VAP to run with the new Raman Lidar at Darwin.

No progress has been made due to other priorities.

Next milestone: Release VAP to run at TWP, and begin routine processing at the DMF by September 30, 2011.

\subsection{Raman Lidar Profiles-MERGE (RLPROFMERGE)}

Translator: Connor Flynn, Pacific Northwest National Laboratory

Developer: Chitra Sivaraman, Pacific Northwest National Laboratory 
Status: Operational

Tier: Production

Engineering Change Order-13736 has been approved to make changes to the VAP to run with the new Raman Lidar at Darwin and fix the handling of glue co-efficients.

The VAP has been released to the DMF for routine processing.

\subsection{Raman Lidar Profiles-Mixing Ratio (RLPROFMR)}

Translator: Connor Flynn, Pacific Northwest National Laboratory

Developer: Chitra Sivaraman, Pacific Northwest National Laboratory

Status: Operational

Tier: Production

Engineering Change Order-13736 has been approved to make changes to the VAP to run with the new Raman Lidar at Darwin.

No progress has been made due to other priorities.

Next milestone: Release VAP to run at TWP, and begin routine processing at the DMF by September 30, 2011.

\subsection{Radiatively Important Parameters Best Estimate (RIPBE)}

Translator: Sally McFarlane, Pacific Northwest National Laboratory

Developer: Tim Shippert, Pacific Northwest National Laboratory

Status: Operational

Tier: Evaluation

The ECO-00767 has been approved to fix bugs and enhance the product based on feedback from beta users.

The RIPBE/BBHRP interface is now available for SGP from March 2002-February 2006 in the evaluation area.

Next milestone: Run RIPBE VAP with the latest MICROBASE data for the four years at SGP. 


\subsection{SGP Area Surface Cloud and SW Radiation Grid (SFCCLDGRID)}

Translator: Sally McFarlane, Pacific Northwest National Laboratory

Developer: Krista Gaustad, Pacific Northwest National Laboratory

Status: Operational

Tier: Production

There are no open ECOs for this VAP.

\subsection{Surface Spectral Albedo (SURFSPECALB)}

Translator: Sally McFarlane, Pacific Northwest National Laboratory

Developer: Krista Gaustad, Pacific Northwest National Laboratory

Status: Operational

Tier: Production

Engineering Change Order-00525 has been approved to review data, address quality check review, automate a test script, and prepare a technical report.

The technical report has been completed, and the VAP has been released to DMF for routine processing.

Historical data have been processed and archived.

Next milestone: Begin routine processing at the DMF.

\subsection{SONDE Adjust (SONDEADJUST)}

Translator: Mike Jensen, Brookhaven National Laboratory

Developer: David Troyan, Brookhaven National Laboratory

Status: In Development

Tier: Evaluation

Engineering Change Order-00824 has been approved to correct the documented biases in radiosonde humidity measurements.

The data have been staged in the evaluation area for all AMF sites and permanent ARM research sites. 


\subsection{Shortwave Flux Analysis (SWFLUXANAL)}

Translator: Sally McFarlane, Pacific Northwest National Laboratory

Developer: Krista Gaustad, Pacific Northwest National Laboratory

Status: Operational

Tier: Production

There are no open ECOs for this VAP.

\subsection{Tower Water-Vapor Mixing Ratio (TWRMR)}

Translator: Sally McFarlane, Pacific Northwest National Laboratory

Developer: Krista Gaustad, Pacific Northwest National Laboratory

Status: Operational

Tier: Production

There are no open ECOs for this VAP.

\subsection{Variational Analysis (VARANAL)}

Translator: Shaocheng Xie, Lawrence Livermore National Laboratory

Developer: Renata McCoy, Lawrence Livermore National Laboratory

Status: Operational

Tier: Evaluation

Engineering Change Request-0096 (ECR-0096) has been approved to develop continuous large-scale forcing data.

Significant progress has been made to produce the ensemble large-scale forcing data for AMF China.

Next milestone: Release the data for AMF China.

\subsection{W-Band ARM Cloud Radar Active Remote Sensing of Clouds (WACRARSCL)}

Translator: Mike Jensen, Brookhaven National Laboratory

Developer: David Troyan, Brookhaven National Laboratory 
Status: Operational

Tier: Evaluation

Engineering Change Request-00826 has been approved to run WACRARSCL at all AMF deployments and continue development at SGP.

All data have been placed at the evaluation area for the AMF sites.

Next milestone: Complete development of WACRARSCL for SGP.

\subsection{Future VAPs}

This section describes new activities that have been approved in the last quarter by the ARM Science and Infrastructure and Science Steering Committee. Work on these activities will begin in the next quarter.

No new VAPs have been approved in the last quarter, but white papers are in the initial stage for Vertical Velocity Stratiform VAP, KAZR-VAP, Precipitation Radar VAP, and SACR-Mapped Moments VAP.

\subsection{Other VAP-Related Work}

\subsection{Pre-Mapping Corrections to SAPR Data}

Translator: Scott Collis, Argonne National Laboratory

Developer: Scott Collis, Argonne National Laboratory

Engineering Work Order-13725 has been initiated to track the process of pre-mapping corrections that need to be performed on the new radar data. This EWO covers work involved in the research and implementation of standard algorithms on the SAPR data for the purposes of generating evaluation data sets.

\subsection{CARES Data Set}

Translator: Jerome Fast, Pacific Northwest National Laboratory

Developer: Chen Song and Manish Shrivastava, Pacific Northwest National Laboratory

Engineering Work Order-13683 has been approved to create an evaluation product from the CARES data set for the Aerosol Modeling Testbed. For details on this work, please visit https://wiki.arm.gov/bin/view/Engineering/VAPWhitePapers. 


\subsection{VAP Metrics}

This section lists the top five VAPs that were requested by users from the Data Archive during the third quarter.

\begin{tabular}{|c|c|c|c|}
\hline Instrument Class & $\begin{array}{c}\text { Number of files } \\
\text { requested }\end{array}$ & $\begin{array}{c}\text { Number of unique } \\
\text { requests }\end{array}$ & $\begin{array}{c}\text { Number of } \\
\text { unique users }\end{array}$ \\
\hline ARSCL & 223870 & 274 & 87 \\
\hline MFRSR* & 182027 & 99 & 60 \\
\hline QCRAD & 118458 & 176 & 96 \\
\hline MWRRET $^{*}$ & 99751 & 102 & 55 \\
\hline$M^{*}$ & 66555 & 137 & 72 \\
\hline
\end{tabular}

* indicates multiple datastreams are included in an instrument class.

\begin{tabular}{|c|c|}
\hline Evaluation data product & $\begin{array}{c}\text { Number of files } \\
\text { requested }\end{array}$ \\
\hline MICROBASE & 11702 \\
\hline MERGESONDE & 7231 \\
\hline MPLCOD & 3274 \\
\hline WACRARSCL & 1582 \\
\hline CLDCLASS & 1068 \\
\hline
\end{tabular}

\begin{tabular}{|c|c|}
\hline Instrument Class & Datastreams \\
\hline MFRSR & MFRSRAOD1MICH \\
\hline & MFRSRCLDOD \\
\hline MPL & MPLCMASK \\
\hline & MPLNOR (Retired) \\
\hline & MPLAVG \\
\hline & MPLSMASK1CLOTH \\
\hline
\end{tabular}




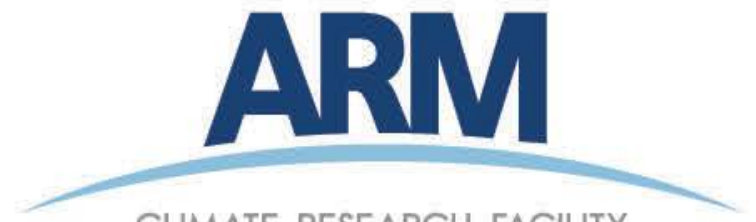

CLIMATE RESEARCH FACILITY

www.arm.gov

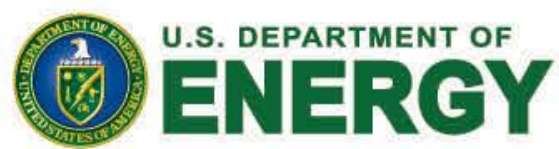

Office of Science 